\title{
Golay improvement of the robustness of mean scatterer spacing measurement with ultrasonic backscattering
}

\author{
Wenlei Pan ${ }^{*}$, Yi Shen, Ting Liu and Yan Wang \\ Department of Control Science and Engineering, Harbin Institute of Technology, Harbin, China
}

\begin{abstract}
Mean scatterer spacing (MSS) estimated from ultrasonic backscattering is of valuable information for tissue characterization. However, low ultrasound frequency, sound attenuation, and diffuse scattering significantly disturb the current MSS measurement methods. The aim of this study is to improve MSS measurement with Coded Excitation (CE) enhanced cepstrum estimation. The study proposes a Golay code-based cepstrum estimation and uses an equivalent Faran cylinder model of cancellous bone. By solving the elastic wave equation, ultrasonic backscattering signals were obtained through simulations. The adopted ultrasonic excitation is $0.9 \mathrm{MHz}$ and is coded with 4-bit complementary sequences, which is modulated by employing a sinusoidal signal. Also, white Gaussian noise of $60 \mathrm{~dB}, 55 \mathrm{~dB}$, and $45 \mathrm{~dB}$ was added to the backscattering data to study the robustness of the proposed method. Simulation results show that the Golay-based method successfully improved ultrasound energy transmission into the tissue and effectively suppressed interference peaks while identifying the right peaks with permitted errors. In addition, the robustness against noise was enhanced.
\end{abstract}

Keywords: Golay codes, mean scatterer spacing, cepstrum estimation, ultrasonic backscattering

\section{Introduction}

Tissues are usually composed of collagen fibers and fibrils, which are quasi-periodical and can be regarded as distributing scatterers. Most of the existing models in tissue engineering [1] for classification and subsequent identification of tissue are based on this hypothesis. Mean Scatterer Spacing (MSS) in tissue can be used to detect diffuse liver disease, hepatic vein thickness [2] and most importantly, cancellous bone trabecular spacing [3]. By measuring the bone's MSS, the microstructure of trabeculae can be characterized and moderate diagnoses of osteoporosis can be made.

Ultrasound is a fundamental method for MSS measurement of the tissue. There are two ways in which the sound waves are scattered. The first is randomly situated scatterers, which are incoherent. The other is coherent scatterers spaced at regular intervals. These contribute to the estimation of MSS.

Major techniques that have been successfully utilized in MSS estimation are wavelet based technique [4], spectral correlation (SAC) based MSS estimation [5], SSA analysis [6] and inverse filter algorithm [7] etc, among which wavelet based method and SSA could also measure the thickness of

\footnotetext{
* Address for correspondence: Wenlei Pan, Department of Control Science and Engineering, Harbin Institute of Technology, Harbin, China. Tel.: +86 451 86413411; Fax: 86-451-86418378; E-mail: wenlei@hit.edu.cn.
} 
scatterers, and SAC method could have more reliable convergence than conventional cepstrum based method over smaller amounts of data. Reflections from the neighboring scatterers interfere constructively and the effect is manifested as several peaks in spectrum that can be used to estimate MSS. However, it is difficult to estimate MSS from ultrasonic backscattering due to the short sequence of the echo, attenuation, and interference of noise. Some robust techniques have been proposed to improve MSS estimation. For example, Keith Wear [8] successfully used autoregressive cepstral estimation to enhance the spatial resolution and accuracy in the characterization of tissue. Jenho Tsao, et al. [9], developed the periodicity detection technique of Periodic Wavelet Transform (PWT), which can tolerate the influence of diffusive speckle interference.

Spatial resolution and attenuation are two main factors that impact MSS estimation accuracy. The normally used ultrasound frequency in MSS estimation is $0.5 \sim 2 \mathrm{MHz}$ [2-9], with wavelength of about $1.5 \sim 6 \mathrm{~mm}$. However, this is not enough to measure bone trabecular scatter spacing $(0.15 \sim 1.2 \mathrm{~mm})$. Also, for porous tissue like cancellous bone, there is significant noise and attenuation [10], which decreases the effective depth that can be used for MSS estimation. To solve these problems, this study presents the technique of coded excitation (CE) based cepstrum estimation by encoding the incidence wave with the Golay complementary sequences. Complementary Golay codes were first introduced by Marcel. J. E Golay in 1949 [11]. Then actual medical ultrasound devices were designed by means of time-serial Golay code, which increased the penetration to get 30 times more power and obtained higher resolution under the same penetration capability [12]. Base on this advantage, it was widely used to improve the medical image quality by transmitting temporally elongated pulse with finite time-bandwidth product, which shows more promising results [13] compared with Barker codes [14] and $\mathrm{m}$-sequences [15]. Nowadays, this technique is widely used in the synthetic aperture imaging [16], the ophthalmological ultrasound [17], and the wave detection in trabecular and long bones [18, 19]. This is the first study to validate the efficiency of CE in MSS estimation. For clarity, 4-bit Golay codes are used. Theoretical works and simulation demonstrations are presented.

\section{Theoretical development}

\subsection{Cepstrum MSS estimation}

Cepstrum is defined as the Inverse Fourier transform of the real logarithm of the squared magnitude of the Fourier transform of a signal [20].

$$
y_{\text {rceps }}=\operatorname{real}\left(\operatorname{IFFT}\left(\log |F F T(x)|^{2}\right)\right)
$$

Cepstrum analysis method was first suggested by Lizzi, et al. [20] when he tried to estimate the wall thickness of hepatic vein. Then Suzuki et al. adopted the cepstrum to measure scatterer spacing in the liver [21].

In most ultrasounds, the backscattering signal is a superposition of simple echoes from scatterers. For one echo, the backscattered data can be represented as [22]

$$
x(t)=s(t)+\delta \cdot s(t-\tau)
$$

where $\delta$ is the attenuation, $\tau=2 \cdot M S S / V_{s}$ is the time delay, and $V_{s}$ is sound speed. The spectral density of such a signal can be given by 


$$
|X(f)|^{2}=|S(f)|^{2}\left[1+\delta^{2}+2 \delta \cos (2 \pi f \tau)\right]
$$

The spectral density (3) is that of the envelop that is modulated by a periodic function of delay $\tau$. The logarithm can be used to convert the spectrum product into the sum of one constant component and one periodic variable component.

$$
B(f)=\log |S(f)|^{2}+\log \left[1+\delta^{2}+2 \delta \cos (2 \pi f \tau)\right]^{2}
$$

Then, the cepstrum is calculated as follows:

$$
C(t)=\operatorname{IFFT}\left(\log |S(f)|^{2}\right)+\operatorname{IFFT}\left(\log \left[1+\delta^{2}+2 \delta \cos (2 \pi f \tau)\right]^{2}\right)
$$

Here $B(f)$ can be seen as an alternative form of time waves, and the periodic components show up as peaks locating at time $t_{\max }=\tau$ in the cepstrum $C(t)$. Then the MSS can be computed as

$$
M S S=t_{\max } \cdot V_{s} / 2
$$

\subsection{Coded Excitation (CE) based MSS estimation}

Cancellous bone is a network of spongy trabeculae. When an ultrasound propagates through it, there is much reflection and scattering at the solid-liquid interface, which reduces ultrasonic energy and the depth it penetrates into bone.

This work proposes that CE-based cepstrum estimation and corresponding pulse compression are techniques that increase the total transmitted energy by encoding the emission sequence. In this study, we are interested in the Golay complementary pair [23]. Golay complementary sequences are pairs of sequences whose out-of-phase aperiodic autocorrelation coefficients' sum is zero [24]. Golay coded excitation uses a long time sequence to excite the transducer, which increases the acoustic power, signal noise ratio, and ultrasonic resolution. If the coded sequence is $e(t)$, both the transmit and receive impulse response of transducer are $p(t)$, the medium function is $u(t)$, and the function of the decoding algorithm is $e^{\prime}(t)$. After compression with a filter, the backscatter signal from cancellous bone $r(t)$ can be expressed as

$$
r(t)=e(t) * p(t) * u(t) * p(t) * e^{\prime}(t)
$$

which can then be rewritten as

$$
r(t)=\left[e(t) * e^{\prime}(t)\right] * p(t) * u(t) * p(t)
$$

With an ideal filter, after decoding $e(t) * e^{\prime}(t)=\delta(t)$, then

$$
r(t)=\delta(t) * p(t) * u(t) * p(t)
$$

which implies that the performance of $\mathrm{CE}$ is equal to the performance of a single pulse excitation with more power [24] and high resolution.

For cancellous bone, if a matched filter is used to compress the backscattering signals coded by 
Golay sequences, the Golay sequences are $V_{x}(t)=X\left(x_{0}, x_{1}, \ldots, x_{\mathrm{n}-1}\right)$ and $V_{y}(t)=Y\left(y_{0}, y_{1}, \ldots, y_{\mathrm{n}-1}\right)$, the impulse response of transducer is $g(t)$, and bone tissue function is $b(t)$. After matched filter the compressed backscattering signals are

$$
\begin{aligned}
& V_{b, x}(f)=\left[V_{x}(f) G(f)\right] B(f) G(f)\left[V_{x}(f) G(f)\right]^{*}=V_{x}(f) G(f) B(f) V_{x}^{*}(f)|G(f)|^{2} \\
& V_{b, y}(f)=\left[V_{y}(f) G(f)\right] B(f) G(f)\left[V_{y}(f) G(f)\right]^{*}=V_{y}(f) G(f) B(f) V_{y}^{*}(f)|G(f)|^{2}
\end{aligned}
$$

In time domain, the backscattering signals are

$$
\begin{aligned}
& R_{b, x}(t, j)=F^{-1}\left[G(f) B(f)|G(f)|^{2}\right] \otimes \sum_{i=0}^{n-j-1} x_{i} x_{i+j} \\
& R_{b, y}(t, j)=F^{-1}\left[G(f) B(f)|G(f)|^{2}\right] \otimes \sum_{i=0}^{n-j-1} y_{i} y_{i+j}
\end{aligned}
$$

The Golay sequences are complementary, which makes the following expression true:

$$
\sum_{i=0}^{n-j-1} x_{i} x_{i+j}+\sum_{i=0}^{n-j-1} y_{i} y_{i+j}= \begin{cases}2 n & j=0 \\ 0 & j \neq 0\end{cases}
$$

Then the obtained backscattering signal from cancellous bone is

$$
R_{b}(t)=R_{b, x}(t, j)+R_{b, y}(t, j)=F^{-1}\left[G(f) B(f)|G(f)|^{2}\right] \otimes\left(\sum_{i=0}^{n-j-1} x_{i} x_{i+j}+\sum_{i=0}^{n-j-1} y_{i} y_{i+j}\right)= \begin{cases}2 n F^{-1}\left[G(f) B(f)|G(f)|^{2}\right] & j=0 \\ 0 & j \neq 0\end{cases}
$$

The data proves that after matched filter, the backscattering signals coded with Golay can effectively suppress the range side lobe and boost the energy levels of backscattering signals, which will improve the efficiency and accuracy in bone MSS characterization.

\section{Simulation setup}

\subsection{Simulation geometry}

The simulations are based on the principle that sound is mechanical waves, and its propagation results in particle vibration and produces acoustic pressure. In this simulation, the time-domain propagation of compressional waves through a medium is modeled as homogeneous and acoustically linear. The sound pressure is calculated by $p=Z \omega \xi$, where $Z=c_{0} \cdot \rho$ is the acoustic impedance, $c_{0}$ is speed of sound, $\rho$ is the density of medium, $\omega=2 \pi f$ is angular frequency, and $\xi$ is the particle displacement.

Solving the elastic wave equation allows us to determine the acoustic pressure of the whole medium with sound propagation [25]:

$$
\rho \frac{\partial^{2} \mathbf{u}}{\partial t^{2}}=\left(\mu+\eta \frac{\partial}{\partial t}\right) \nabla^{2} \mathbf{u}+\left(\lambda+\mu+\xi \frac{\partial}{\partial t}+\frac{\eta}{3} \frac{\partial}{\partial t}\right) \nabla \nabla \cdot \mathbf{u}
$$


Where $\lambda$ and $\mu$ are Lame constants, $\mathbf{u}=\mathbf{u}(x, y, z, t)$ is displacement field vector, $\eta$ and $\xi$ are viscosity, and $\rho$ is the bone density .

Several models, including the Faran cylinder model, binary fixture model, and weak scattering model [26], are usually used to model the cancellous bone microstructure. This work used the Faran model. Micro-computed tomography (micro-CT) can non-destructively provide information about the tissues' internal structure [27], so this study measured the real bone microstructure with uCT35 (SCANCO Medical AG) and a slice of trabeculae (P0-1), as shown in Figure 1. The scatterer along certain sound paths (Fig. 1(B)) is described by an equivalent scatterer model (Fig. 1(C)).

An anisotropic absorbing boundary was implemented around the domain of interest (DOI) to prevent waves from leaving the boundary sides and being reintroduced into the backscattered waves. Scatterers are lined up and space $4 \mathrm{~mm}$ apart. According to the characteristics of bone material, the density of medium and scatterer are 1300 and $938 \mathrm{~kg} / \mathrm{m}^{3}$ respectively; acoustic velocities are 3300 and $1500 \mathrm{~m} / \mathrm{s}$. The variation of material characteristics along the wave propagation path is shown in Fig. 2. Frequency of ultrasound excitation is $0.9 \mathrm{MHz}$. The simulations were implemented in Matlab R2009a (The Mathworks Inc., Natick, MA).

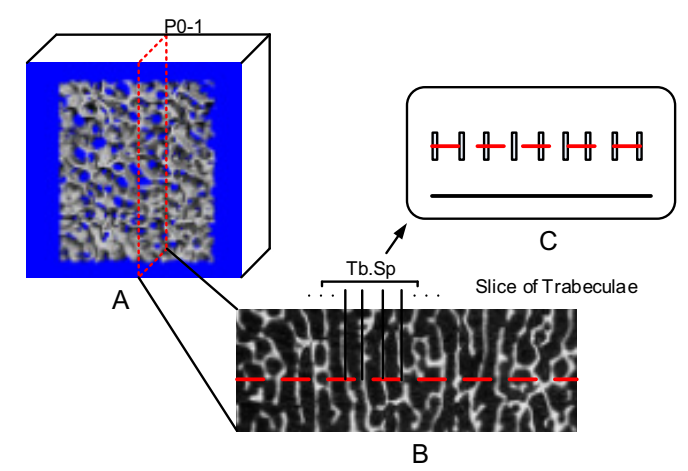

Fig. 1. Computed tomography image of cancellous bone microstructure. (A) Three-dimensional trabeculae; (B) A slice of position P0-1; (C) Equivalent Faran model. It should be noted that the separation distance in Faran model is much smaller than ultrasonic wavelength.
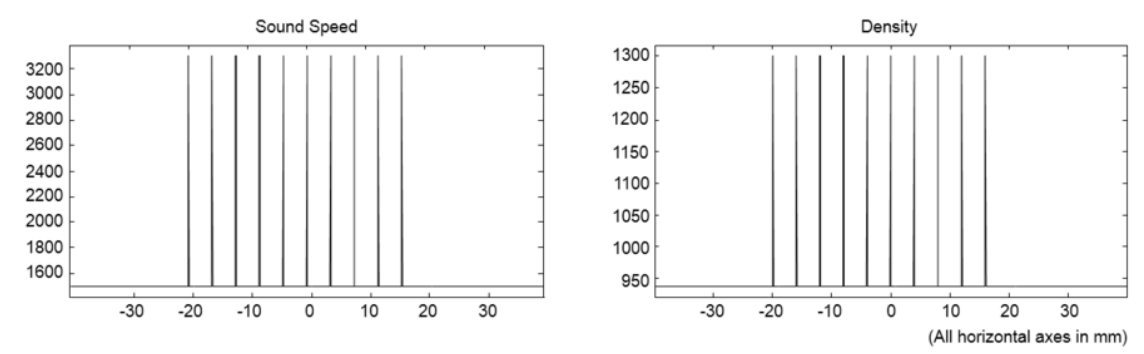

Fig. 2. Distribution of material characteristics along the wave propagation path. 10 scatterers ranges from $-20 \mathrm{~mm}$ to 18 $\mathrm{mm}$ with separation distance of $4 \mathrm{~mm}$. The ultrasound speed and density of scatterers are $3300 \mathrm{~m} / \mathrm{s}$ and $1300 \mathrm{~kg} / \mathrm{m}^{3}$; for the transmission medium, they are $1500 \mathrm{~m} / \mathrm{s}$ and $938 \mathrm{~kg} / \mathrm{m}^{3}$.

\subsection{Application of Golay complementary sequences to cepstrum estimation}

Numerous binary sequences have been used for ultrasonic coded excitation. Some common methods include pseudo-random PN sequences, Chirp codes, Golay codes, and Golay complementary pair [28]. In our ultrasonic bone system, the length of coded waves should be kept short and the sequences 
should be easy to modulate within capacity of the hardware terminal. Our previous research showed that the Golay method works better. In deep structure ultrasound imaging, with Golay coding, the axial resolution of fundamental and third harmonic components were calculated to be $0.91 \mathrm{~mm}$ and $0.7 \mathrm{~mm}$ respectively, which were 0.6 and 0.46 times the values obtained with conventional pulsing using the same transducer [29]. With high ultrasound frequency of $10 \mathrm{MHz}$ (100 MHz sampling rate), the error of displacement measurements was $-5.76 \mathrm{~nm} \pm 36.27 \mathrm{~nm}$ [30], while the resolution of the traditional pulse echoes system, using the same transducer and sampling rate, is about $7.5 \mathrm{um}[31,32]$.

In this study, 4-bit Golay pairs, which are composed of two 4-digit binary sequences, were used. The sequence pair is constructed as follows: if the n-digit binary complementary pair of codes are $A[n]$ and $\mathrm{B}[\mathrm{n}]$, then a new pair of complementary codes with length of $2 \mathrm{n}, \mathrm{A}^{\prime}[2 \mathrm{n}]$, and $\mathrm{B}^{\prime}[2 \mathrm{n}]$ can be constructed by concatenating the original pair $\{\mathrm{A}[\mathrm{n}] \mid \mathrm{B}[\mathrm{n}]\} \quad\{\mathrm{A}[\mathrm{n}] \mid \sim \mathrm{B}[\mathrm{n}]\}$, where $\sim \mathrm{B}[\mathrm{n}]$ is the complement of $\mathrm{B}[\mathrm{n}]$. It is easy to confirm that $\mathrm{A}[2]=[+1+1]$ and $\mathrm{B}[2]=[+1-1]$ are a pair of complementary codes; therefore, the 4-digit codes used in this work are established as $A[4]=[+1+1+1$ $-1]$ and $\mathrm{B}[4]=[+1+1-1+1]$.

Our simulation modulated the binary sequences before exciting the probe, so the spectra of Golay sequences were well matched to the transducer bandwidth [28]. A sinusoidal signal was used where each positive $(+1)$ or negative $(-1)$ symbol was modulated. The sinusoidal signal was located at the center frequency of the excitation (fp), as shown in Fig. 3. In Golay sequences, the element +1 corresponds to a sine wave with the phase of 0 , and -1 corresponds to a symbol at the phase of $\pi$.

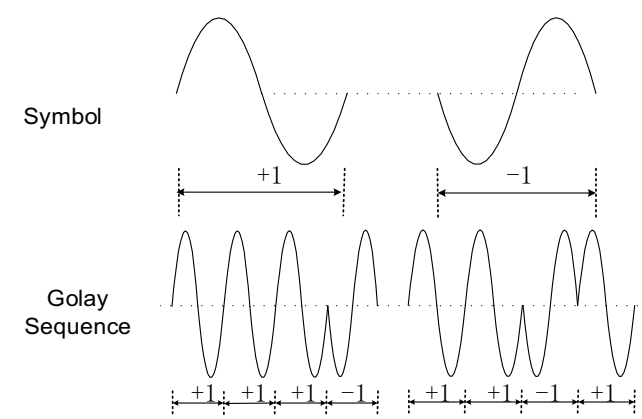

Fig. 3. Example of the modulation method of Golay codes and Golay sequence pair $[+1+1+1-1][+1+1-1+1]$.

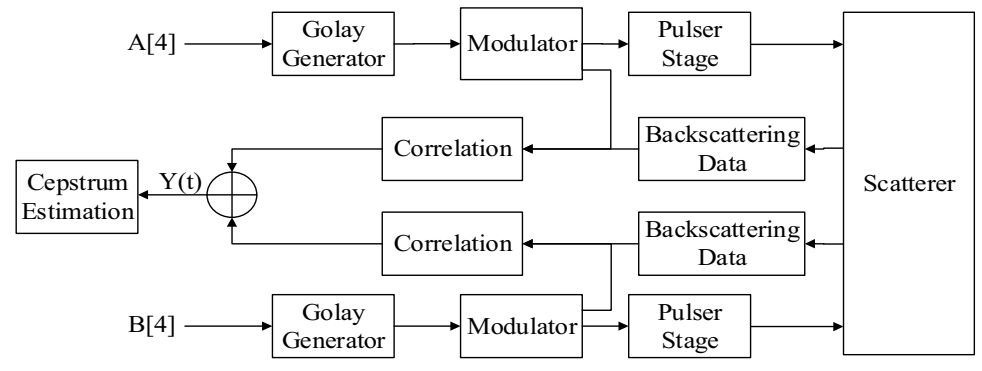

Fig. 4. Diagram of Golay code based cepstrum estimation. 


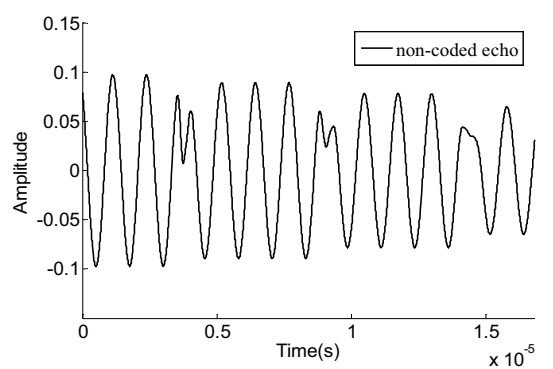

(A)

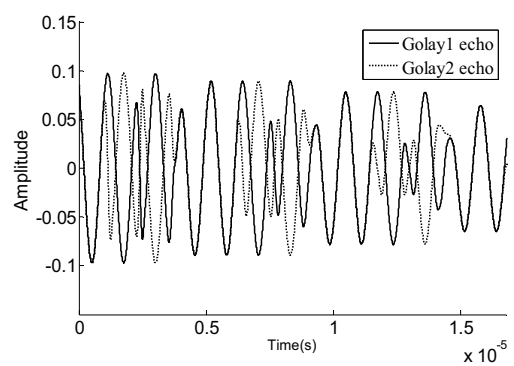

(B)

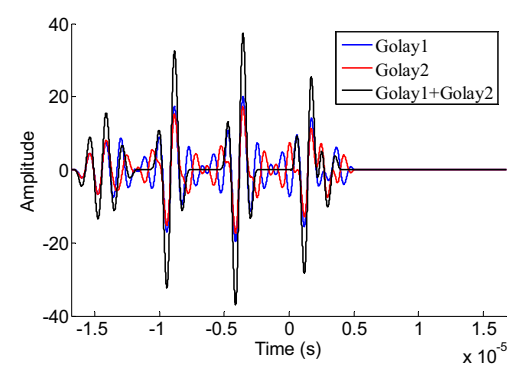

(C)

Fig. 5. Ultrasonic backscattering signals without the influence of noise. (A) Signals unenhanced; (B) Signals enhanced with CE; (C) Compressed signal after decoding.

The excitation pulse in a coded system extends over a longer period of time than impulse response of transducers, which then were compressed by correlating the received backscattering data with reference waveforms to yield signals that approximate the impulse response of a normal pulsed system. Fig. 4 shows the diagram of CE based estimation, in which the incident wave works as the reference waveform. Before exciting the pulser, the Golay sequence pairs were generated and modulated. Then correlations were made between the modulated incident data and the backscattering data from scatterers for each sequence as matched filtering. Correlation results of the two sequences were summed to obtain the final backscattering data, which were then used for MSS cepstrum estimation.

\section{Results and discussion}

This work studied CE enhanced MSS estimation by using Golay complementary sequences to encode the incident wave and improve the performance of cepstrum estimation with ultrasonic backscattering. The ultrasonic wave propagation and backscattering from medium of scatterers were simulated with the elastic wave equation.

Ultrasonic backscattering signals are shown in Fig. 5, where (A) is the signal without coded excitation; (B) is the two backscattering signals coded with Golay $1[+1+1+1-1]$ and Golay $2[+1+1$ $-1+1]$; and $(\mathrm{C})$ is the decoding results of each coded transmitting wave and its corresponding backscattering signal. The selected data length is $15.7 \mathrm{us,} \mathrm{which} \mathrm{corresponds} \mathrm{to} \mathrm{the} \mathrm{propagation}$ distance of $25 \mathrm{~mm}$. The limited distance of sound propagation strictly limits attenuation. After correlation decoding, the side lobes of the constructed Golay pairs proved to be complementary with the opposite amplitudes, while the main lobes are cophasal and redoubled after summation operation. This comes from the auto-correlation functions of A [4] and B [4] in (14). Assuming that the medium system can be expressed as $M_{s}$, decoding the backscattering data $A_{44}(t) * M_{S} * A_{44}(-t)+B_{47}(t) * M_{S} * B_{47}(-t)=M_{S} *\left(E\left(A_{44}\right)+E\left(B_{47}\right)\right)$ benefits from the complementarity of sequence pairs A/B. This contributes to the above suppressed side lobes and redoubled main lobes and allows for more ultrasound energy and deeper penetration in bone tissue.

Generally, the existence of diffuse scattering causes several cepstrum peaks to appear in MSS measurement, but only one of them is the right peak and needs to be enhanced and identified. Fig. 6 shows the MSS estimation results with non-enhanced cepstrum and the Golay based cepstrum method. The results of the cepstrum estimation without coding showed an interference peak, which is highest but not the expected. However, for the Golay enhanced cepstrum method, the highest peak appeared at the right distance of $3.838 \mathrm{~mm}$, approximate to the right $\mathrm{MSS}=4 \mathrm{~mm}$, with errors permitted in tissue 


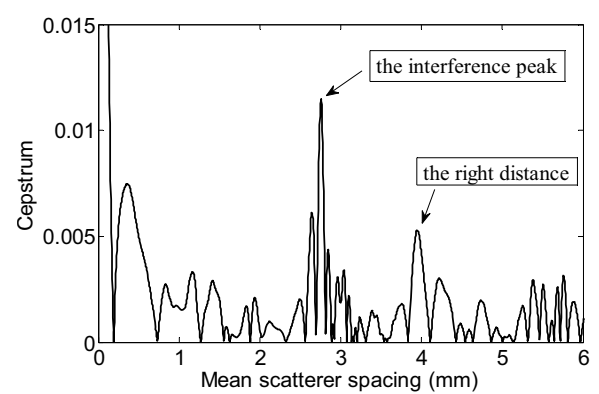

(A)

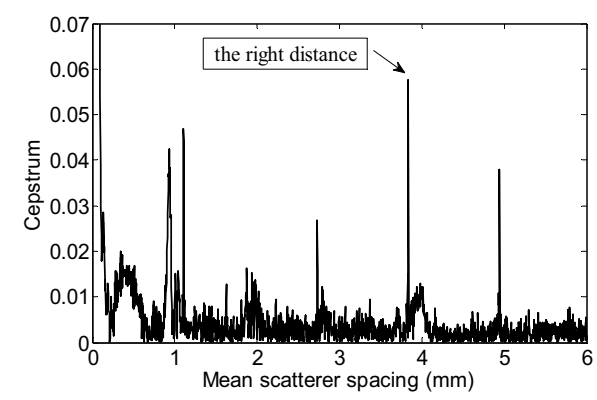

(B)

Fig. 6. Non-enhanced Mss estimation (A) and Golay-enhanced MSS estimation (B) with ultrasonic backscattering.

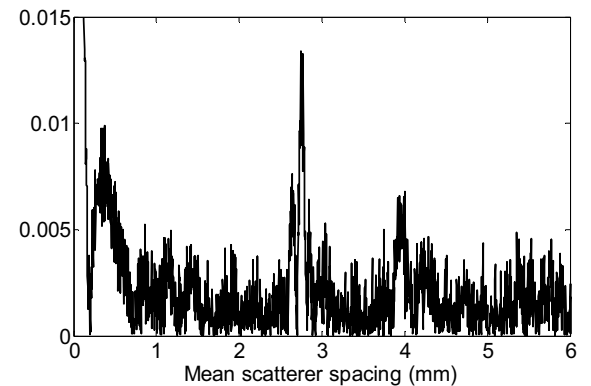

(A)

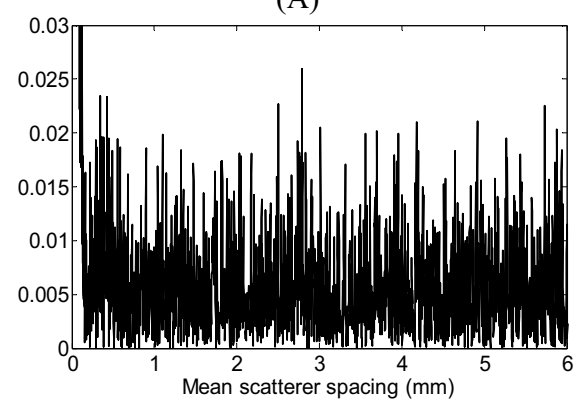

(C)

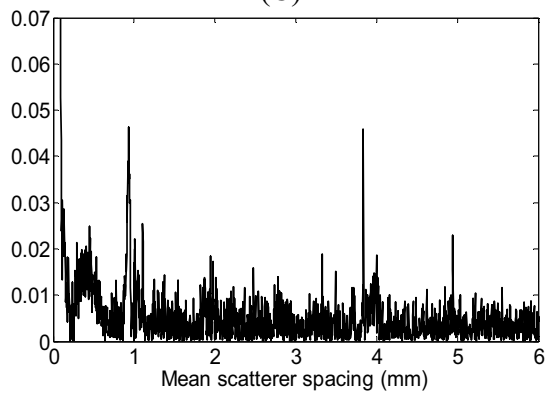

(E)

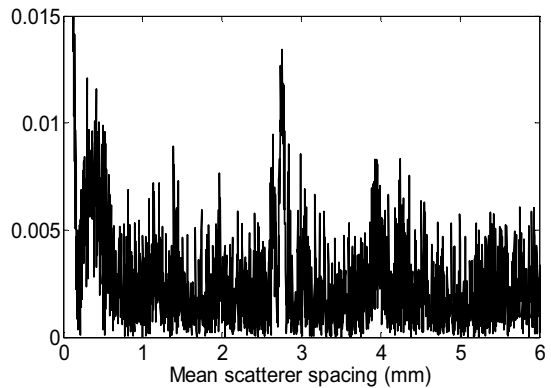

(B)

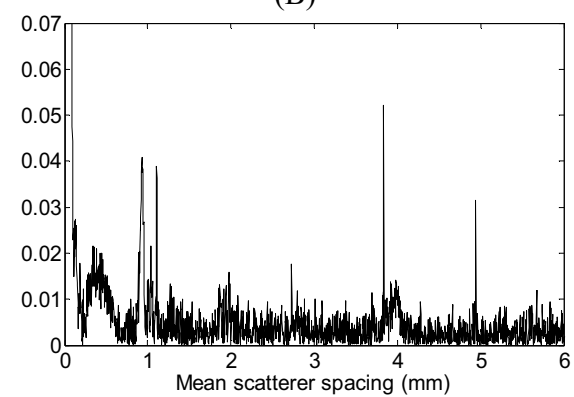

(D)

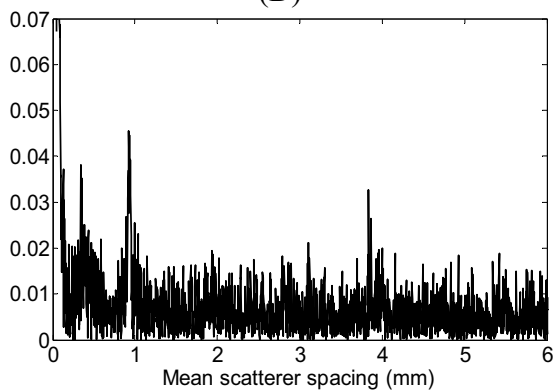

(F)

Fig. 7. Mss estimation from backscattering signals with noise $S N R=60 \mathrm{~dB}$ in (A) \& (D), $\mathrm{SNR}=55 \mathrm{~dB}$ in (B) \& (E), $\mathrm{SNR}=45 \mathrm{~dB}$ in $(\mathrm{C}) \&(\mathrm{~F})$. Among these backscattering data, (D), (E) and (F) are Golay enhanced.

characterization. For bone tissue, the error comes from the scatterer thickness, which is about $0.05 \mathrm{~mm}$ with sound speed of $3000 \mathrm{~m} / \mathrm{s}$, higher than that of the medium $(1500 \mathrm{~m} / \mathrm{s})$. Compared to the non-enhanced method, for instantaneous peaks with the same power value, the SNR in Golay based 
MSS estimation is improved by a factor approximate to the time-bandwidth product of the CE sequence. This improvement also comes from the side lobe suppression discussed above, in which the MSS estimation makes the coded backscattering data perform like backscattering signals from a single narrow incident wave.

To further demonstrate the effect of noise on MSS estimation, we added white Gaussian noise of 60 $\mathrm{db}, 55 \mathrm{db}$, and $45 \mathrm{db}$ to the backscattering signals. The results demonstrated that without $\mathrm{CE}$, the main cepstrum peaks were intensely disturbed (Figure 7(A)-7(C)), which make it difficult to make the right estimation when the SNR increased to $45 \mathrm{~dB}$. However, when the incident ultrasonic waves were enhanced with Golay codes, the robustness of MSS estimation was significantly improved. For example, when $\mathrm{SNR}=60 \sim 55 \mathrm{~dB}$, there was almost no influence to the cepstrum peaks in MSS measurement compared with Fig. 6(B). When SNR increased to $45 \mathrm{~dB}$, the right cepstrum peak was disturbed but still obvious enough for scatterer estimation. Here it should be noted that at higher noise level of $55 \mathrm{~dB}$ and $45 \mathrm{~dB}$, the peaks at $1 \mathrm{~mm}$ are almost the same and even higher than the right peaks at $4 \mathrm{~mm}$, which implies that the algorithm in this work is limited if there is too much noise. In real tissue examining, to make the right peaks rationally picked, further research on the noise reduction especially in vivo measurement are needed.

For ultrasonic medical in vivo measurement, a strict requirement for the stability of the ultrasound probe locations when stimulating the 1st and 2 nd Golay coded waves are needed. This is difficult to achieve and any deviation will cause mismatch between two backscattered waves and then weaken the performance enhancement. In addition, in this work, we used a 4-bit code to improve the MSS estimation results. A longer coded incident wave (for 4-bit, the length of the incident wave is $12 \mathrm{~mm}$ when the sound speed is $1500 \mathrm{~m} / \mathrm{s}$ in water) will cause a significant overlap between the incident and backscattered echoes; and limitations of the circuit power and the requirement of piezoelectricity matching will make it a challenge to stimulate and collect longer codes for an ultrasonic system.

\section{Conclusion}

This work proposed a Golay code based cepstrum estimation method to validate CE enhancement in measuring MSS with ultrasonic backscattering. The coded backscattering data was obtained through simulations from an equivalent Faran cylinder model. Results suggest that Golay codes are capable of effectively improving the performance of MSS estimation. If the period of the excitation pulse is extended, and then received backscattering data is compressed through correlation with reference waveforms, then suppressed side lobe and redoubled main lobes are obtained, which implies longer penetration depth in tissue. In addition, interference peaks in cepstrum estimation are suppressed and the peaks corresponding to the right MSS are identified; the robustness against noise is also enhanced.

Increasing tissue penetration while preserving fine range resolution is rather contradicting demands in ultrasonography and inner tissue structure measurement. The sound energy absorption in tissue increases with frequency when examining the deep structure. How to efficiently enlarging the penetration, improve SNR and increase axial resolution with Golay codes is under research. In addition, the development and implementation of hardware is now being investigated so that it allows for the excitation of long Golay codes. Our future research will focus on the study of CE enhanced measurement with long code sequences and frequency modulation methods, including its efficiency and applicability in vivo measurement. 


\section{Acknowledgment}

This work was supported by Program for Interdisciplinary Basic Research of Science-Engineering-Medicine in Harbin Institute of Technology.

\section{References}

[1] J. Liu, G. Li, L. Ren, et al., Microstructure and mechanical properties of polycaprolactone/calcium sulfate particle and whisker composites, 2012 International Conference on Biomedical Engineering and Biotechnology (iCBEB), Macau, China, 2012, pp. 1558-1561.

[2] R.S. Mia, M.H. Loew, K.A. Wear, et al., Quantitative estimation of scatterer spacing from backscattered ultrasound signals using the complex cepstrum, Information Processing in Medical Imaging, Springer Berlin Heidelberg, 1997, pp. 513-518.

[3] K. Huang, D. Ta, W. Wang, et al., Simplified inverse filter tracking algorithm for estimating the mean trabecular bone spacing IEEE Transactions on Ultrasonics, Ferroelectrics and Frequency Control 55 (2008), 1453-1464.

[4] X. Tang and U.R. Abeyratne, Wavelet transforms in estimating scatterer spacing from ultrasound echoes Ultrasonics 38 (2000), 688-692.

[5] T. Varghese and K.D. Donohue, Mean-scatterer spacing estimates with spectral correlation The Journal of the Acoustical Society of America 96 (1994), 3504-3515.

[6] W.C.A. Pereira, S.L. Bridal, A. Coron, et al., Singular spectrum analysis applied to backscattered ultrasound signals from in vitro human cancellous bone specimens, IEEE Transactions on Ultrasonics, Ferroelectrics, and Frequency Control 51 (2004), 302-312.

[7] K. Huang, D. Ta, W. Wang, et al., Simplified inverse filter tracking algorithm for estimating the mean trabecular bone spacing IEEE Transactions on Ultrasonics, Ferroelectrics, and Frequency Control 55 (2008), 1453-1464.

[8] K.A Wear, R.F. Wagner, M.F. Insana, et al., Application of autoregressive spectral analysis to cepstral estimation of mean scatterer spacing IEEE Transactions on Ultrasonics, Ferroelectrics and Frequency Control 40 (1993), 50-58.

[9] T. Jenho and S. Guang, Mean scatterer spacing estimation using wavelet spectrum, Ultrasonics Symposium 3 (2014), 2090-2093.

[10] S. Tsao and J. Tsao, An unbiased tissue attenuation coefficient estimator using microbubble echoes with harmonic ratio compensation, 2012 International Conference on Biomedical Engineering and Biotechnology (iCBEB), Macau, China, 2012, pp. 727-730.

[11] M. E. Golay, Multi-slit spectrometry Journal of the Optical Society of America 39 (1949), 437-437.

[12] Y. Takeuchi, An investigation of a spread energy method for medical ultrasound systems: Part two: Proposed system and possible problems Ultrasonics 17 (1979), 219-224.

[13] C. Jin, S. Chen and T. Wang, A new scheme of coded ultrasound using Golay codes Journal of Zhejiang University Science C 11 (2010), 476-480.

[14] H. Zhao, L.Y.L. Mo and S. Gao, Barker-coded ultrasound color flow imaging: theoretical and practical design considerations, IEEE Transactions on Ultrasonics, Ferroelectrics and Frequency Control 54 (2007), 319-331.

[15] J. Shen and E.S. Ebbini, A new coded-excitation ultrasound imaging system I. Basic principles, IEEE Transactions on Ultrasonics, Ferroelectrics and Frequency Control 43 (1996), 131-140.

[16] M. Yang and C. Chakrabarti, Design of orthogonal coded excitation for synthetic aperture imaging in ultrasound systems, 2012 IEEE International Symposium on Circuits and Systems (ISCAS), Seoul, Korea, 2012, pp. 113-116.

[17] S. Zhou, X. Wang, J. Ji, et al., Ophthalmological ultrasound biometer using Golay-coded pulse excitation Biomedical, 2014 7th International Conference on Engineering and Informatics (BMEI), Dalian, China, 2014, pp. 76-80.

[18] B. Lashkari, A. Manbachi, A. Mandelis, et al., Slow and fast ultrasonic wave detection improvement in human trabecular bones using Golay code modulation The Journal of the Acoustical Society of America 132 (2012), EL222-EL228.

[19] H. Zhang, S. Wu, D. Ta, et al., Coded excitation of ultrasonic guided waves in long bone fracture assessment Ultrasonics 54 (2014), 1203-1209.

[20] F. Lizzi, E. Feleppa and N. Jaremko, Liver-tissue characterization by digital spectrum and cepstrum analysis 1981 Ultrasonics Symposium, 1981, pp. 575-578.

[21] T. Kamada, Ultrasonic tissue characterization of chronic liver disease using cepstral analysis Gastroenterology 101 (1991), 1325-1331.

[22] A.V. Oppenheim and R.W. Schafer, From frequency to quefrency: A history of the cepstrum Signal Processing Magazine 21 (2004), 95-106. 
[23] Z. Luo, J. Zheng, J. Zhu, et al., Golay complementary pair aided time synchronization method for OFDM systems, 2012 IEEE 14th International Conference on Communication Technology (ICCT), 2012, pp. 166-170.

[24] X. Song, D. Ta and W. Wang, A base-sequence-modulated golay code improves the excitation and measurement of ultrasonic guided waves in long bones, IEEE Transactions on Ultrasonics, Ferroelectrics and Frequency Control 59 (2012), 2580-2583.

[25] J.L. Rose and P.B. Nagy, Ultrasonic waves in solid media The Journal of the Acoustical Society of America 107 (2000), 1807.

[26] K.A. Wear, Ultrasonic scattering from cancellous bone: A review, IEEE Transactions on Ultrasonics, Ferroelectrics and Frequency Control 55 (2008), 1432-1441.

[27] J. Fu and R. Tan, In-line phase contrast micro-CT reconstruction for biomedical specimens Bio-Medical Materials and Engineering 24 (2014), 431-437.

[28] R.M. Garcia, Y. Yañez, H.M. Garcia, et al., Application of Golay codes to improve the dynamic range in ultrasonic Lamb waves air-coupled systems NDT \& E International 43 (2010), 677-686.

[29] W.R. Ridgway, Deep structure ultrasound imaging using fundamental and third harmonic coded excitation techniques, Master Thesis, University of Illinois at Urbana-Champaign, 2012.

[30] Q. Peng and L.Q. Zhang, High-resolution ultrasound displacement measurement using coded excitations, IEEE Transactions on Ultrasonics, Ferroelectrics and Frequency Control 58 (2011), 122-133.

[31] M.F. Insana, L.T. Cook, M. Bilgen, et al., Maximum-likelihood approach to strain imaging using ultrasound The Journal of the Acoustical Society of America 107 (2000), 1421-1434.

[32] Y.P. Zheng, S.L. Bridal, J. Shi, et al., High resolution ultrasound elastomicroscopy imaging of soft tissues: System development and feasibility Physics in Medicine and Biology 49 (2004), 3925. 